\title{
Dark matter deprivation in the field elliptical galaxy NGC $7507^{\star}$
}

\author{
Richard R. Lane ${ }^{1}$, Ricardo Salinas ${ }^{2}$, and Tom Richtler ${ }^{1}$ \\ 1 Departamento de Astronomía Universidad de Concepción, Casilla 160 C, Concepción, Chile \\ e-mail: rlane@astro-udec.cl \\ 2 Department of Physics and Astronomy, Michigan State University, East Lansing, MI 48824, USA \\ Received 25 April 2014 / Accepted 10 December 2014
}

ABSTRACT

\begin{abstract}
Context. Previous studies have shown that the kinematics of the field elliptical galaxy NGC 7507 do not necessarily require dark matter. This is troubling because, in the context of $\Lambda \mathrm{CDM}$ cosmologies, all galaxies should have a large dark matter component. Aims. Our aims are to determine the rotation and velocity dispersion profile out to larger radii than do previous studies, and, therefore, more accurately estimate of the dark matter content of the galaxy.

Methods. We use penalised pixel-fitting software to extract velocities and velocity dispersions from GMOS slit mask spectra. Using Jeans and MONDian modelling, we then produce models with the goal of fitting the velocity dispersion data.

Results. NGC 7507 has a two-component stellar halo, with the outer halo counter rotating with respect to the inner halo, with a kinematic boundary at a radius of $\sim 110^{\prime \prime}(\sim 12.4 \mathrm{kpc})$. The velocity dispersion profile exhibits an increase at $\sim 70^{\prime \prime}(\sim 7.9 \mathrm{kpc})$, reminiscent of several other elliptical galaxies. Our best fit models are those under mild anisotropy, which include $\sim 100$ times less dark matter than predicted by $\Lambda \mathrm{CDM}$, although mildly anisotropic models that are completely dark matter free fit the measured dynamics almost equally well. Our MONDian models, both isotropic and anisotropic, systematically fail to reproduce the measured velocity dispersions at almost all radii.

Conclusions. The counter-rotating outer halo implies a merger remnant, as does the increase in velocity dispersion at $\sim 70^{\prime \prime}$. From simulations it seems plausible that the merger that caused the increase in velocity dispersion was a spiral-spiral merger. Our Jeans models are completely consistent with a no dark matter scenario, however, some dark matter can be accommodated, although at much lower concentrations than predicted by $\Lambda$ CDM simulations. This indicates that NGC 7507 may be a dark matter free elliptical galaxy. Regardless of whether NGC 7507 is completely dark matter free or very dark matter poor, it is at odds with predictions from current $\Lambda \mathrm{CDM}$ cosmological simulations. It may be possible that the observed velocity dispersions could be reproduced if the galaxy is significantly flattened along the line of sight (e.g. due to rotation); however, invoking this flattening is problematic.
\end{abstract}

Key words. galaxies: individual: NGC 7507 - galaxies: kinematics and dynamics - galaxies: elliptical and lenticular, cD galaxies: halos

\section{Introduction}

The existence of dark matter in spiral galaxies, or the possibility of a gravitational interaction which differs from that of Newtonian gravity, is not generally disputed. It seems that central cluster galaxies are also embedded in massive dark haloes (see e.g. Kelson et al. 2002; Richtler et al. 2008, 2011a; Schuberth et al. 2010), as are some of the most massive noncentral galaxies in the Fornax and Virgo clusters (e.g. Napolitano et al. 2011; Schuberth et al. 2012). However, isolated elliptical galaxies (IEs) and galaxies in less dense environments are only beginning to be studied in detail.

As the isolated counterparts to massive ellipticals in clusters, IEs should contain information highlighting their evolutionary differences. Globular cluster (GC) and planetary nebula (PN) populations are now being employed to this end, in particular as dynamical probes of galactic haloes (e.g. Romanowsky et al. 2003; Douglas et al. 2007; Napolitano et al. 2009; Méndez et al. 2009; Teodorescu et al. 2010; Richtler et al. 2011a).

* Based on observations taken at the Gemini Observatory, operated by the Association of Universities for Research in Astronomy, Inc., under a cooperative agreement with the NSF on behalf of the Gemini partnership: the National Science Foundation (United States), the Science and Technology Facilities Council (United Kingdom), the National Research Council (Canada), CONICYT (Chile), the Australian Research Council (Australia), Ministério da Ciência e Tecnologia (Brazil) and SECYT (Argentina).
Techniques for analysing spectra of the stellar halo of galaxies have also been developed over the past decade or so (e.g. Kronawitter et al. 2000; Kelson et al. 2002; Cappellari \& Emsellem 2004; Samurović \& Danziger 2005; Salinas et al. 2012), and more recently in the context of mask spectroscopy (e.g. Norris et al. 2008; Proctor et al. 2009; Foster et al. 2009, $2011,2013)$. Analysis of the stellar halo light of a galaxy has the advantage that, since the diffuse stellar halo is being observed, there are no restrictions on image quality, as is the case for GCs and PNe, while still reaching significantly large radii. The advantage of mask spectroscopy is that spectra can be obtained at a variety of position angles within a single observation.

Interestingly, from studies of GCs and PNe, as well as of galaxy halo light, it appears that not all elliptical galaxies require dark matter (DM) to explain their dynamics, and several lower mass IEs only require a minimal dark halo. This was first investigated at least 20 years ago (Ciardullo et al. 1993; Bertin et al. 1994) and such investigations continue today (de Lorenzi et al. 2009; Morganti et al. 2013). However, numerical simulations based on Lambda cold dark matter $(\Lambda \mathrm{CDM})$, the leading paradigm for Universal large scale structure formation, show that massive galaxies in all environments, including IEs, should be dominated by DM (e.g. Niemi et al. 2010). Therefore, if the paucity of DM in some IEs can be corroborated, and even evidenced in different galactic environments, this could have serious implications for $\Lambda \mathrm{CDM}$ cosmological models, at least in their current form. 
One example of an IE that may not require DM to explain its dynamics is NGC 7507, as shown recently by (Salinas et al. 2012; hereafter S12). Models with a cored logarithmic DM halo could also reproduce the observed velocity dispersion profile, assuming some radial anisotropy, however, these models only contained a maximum $7.4 \%$ of the total mass as DM within 2 effective radii $\left(R_{\mathrm{e}}\right)$. Since the influence of DM in high surface brightness galaxies can only be unmistakably detected at large radii, measuring the galaxy kinematics at these large radii is essential. The very interesting result by S12 has the limitation that the longslit spectra gathered were only able to probe to radii $R \lesssim 1 R_{\mathrm{e}}$.

In the current paper we extend the work of S12 using GMOS slit masks to cover a large range of position angles and obtain a velocity field out to $\sim 195^{\prime \prime}, \sim 2.6, R_{\mathrm{e}}\left(\mathrm{S} 12: R_{\mathrm{e}} \sim 75^{\prime \prime}\right)$. This allows us to search for rotation signatures of the halo out to large radii (Sect. 3), as well as extend the velocity dispersion profile out to $\sim 1.7 R_{\mathrm{e}}$ (Sect. 4 ). Furthermore, with the velocity dispersion profile we were able to produce dynamical models out to larger radii than previously possible, to probe the dark matter content of the galaxy (Sect. 5). Note that our assumed distance to NGC 7507 of $23.22 \pm 1.8 \mathrm{Mpc}$ (Tonry et al. 2001; corrected by -0.16 mag as per Jensen et al. 2003), implies a scale of $112.5 \mathrm{pc}$ per arcsecond, which we will use throughout this paper.

\section{Observations and data reduction}

Observations were taken during the night of September 16, 2009, in queue mode, using the Gemini Multi-Object Spectrograph (GMOS) on the Gemini south telescope, at Cerro Pachón, Chile (Gemini Program GS-2009B-Q-84), during dark time (4\% Lunar illumination). A single mask with $1^{\prime \prime}$ slit widths, centred on NGC 7507, was used with the B600+_G532 grism, giving a resolution of $\sim 4.7 \AA F W H M$, for consistency with the long-slit study by S12. Slit lengths (4.'5-8.'0) and positions were chosen such that no known objects overlapped any slit, to ensure as pure a galactic stellar population as possible within the slits. Figure 1 shows the slit positions of the 47 slits on the slit-mask, overlaid on the GMOS field of view.

Eight exposures of 1700 s were separated by $2 \times 1700$ s sky exposures, at a blank sky position $13^{\prime}$ south of the galaxy, and four $\mathrm{Cu}-\mathrm{Ar}$ arc exposures, for use in reduction and calibration. This gave us a total exposure time of $3 \mathrm{~h}$ and $47 \mathrm{~min}$, on source. The sky exposures were taken immediately following the science exposures to ensure changes in sky illumination were minimised. The maximum time between science and sky exposures was $\sim 1$ min. Having the blank sky position 13' from the source precluded galaxy light from contaminating our sky spectra. Furthermore, this also removes the necessity to invoke complex algorithms for removing the sky background (e.g. Norris et al. 2008) or rely on slits at large radii which still contain some galaxy light (e.g. Proctor et al. 2009).

The 47 slits were concentrated on the outer parts of the galaxy, where a low surface brightness, and hence, a low signal is expected. Because of this, the reduction process should, ideally, minimise the introduction of noise. The 8 science frames were median combined directly as 2D frames using the IRAF/Gemini routine gemcombine. The two sky frames were averaged together, also as $2 \mathrm{D}$ frames, and this "master" sky frame was subtracted from the median science frame. This also removed the bias, without further introduction of noise. The data from the three GMOS detectors were then mosaiced using the IRAF/Gemini routine gmosaic. The combined and sky subtracted frame was then wavelength calibrated using

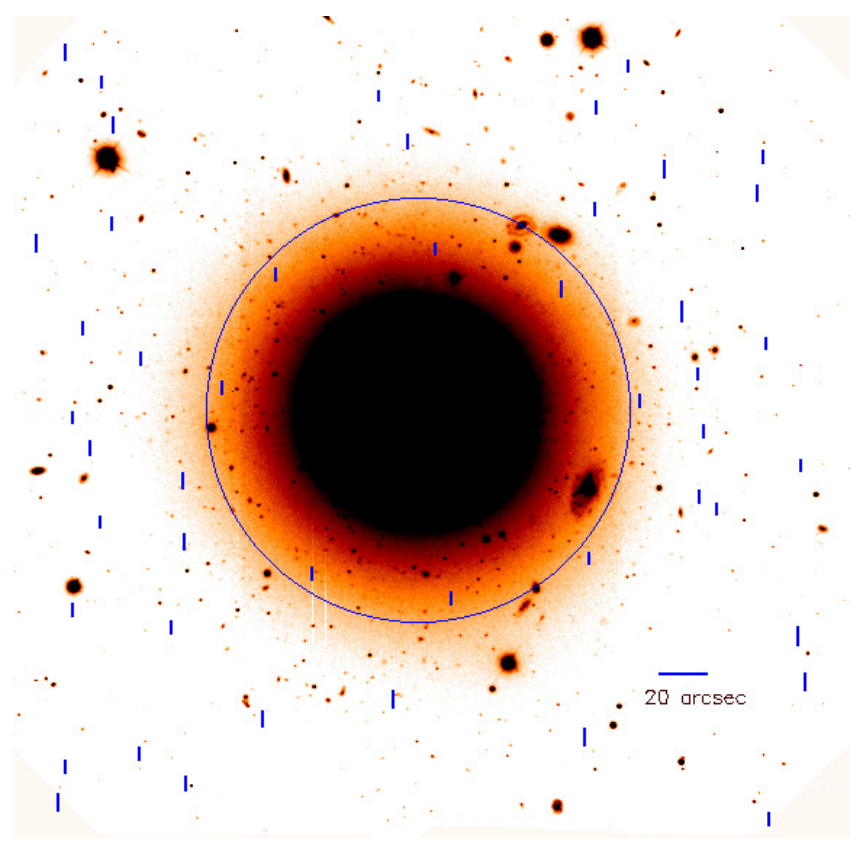

Fig. 1. GMOS mask slit positions, centred on NGC 7507, at $(\mathrm{RA}, \mathrm{Dec})=\left(23^{\mathrm{h}} 12^{\mathrm{m}} 07^{\mathrm{s}},-28^{\circ} 32^{\prime} 23^{\prime \prime}\right)$. The science slit positions and lengths $\left(4{ }^{\prime \prime} 5-8{ }^{\prime \prime} 0\right)$, are represented as vertical blue lines. All slits are $1^{\prime \prime}$ in width. The circle indicates the largest radius probed by S12. The GMOS field of view is $\sim 5.5 \times 5.5$, and a $20^{\prime \prime}$ bar is shown for scale. North is down and east is to the left.

the $\mathrm{Cu}-\mathrm{Ar}$ arc taken after the science exposures, with the aid of the IRAF task gswavelength. The typical RMS of the dispersion solution was $\sim 0.06 \AA$. This dispersion solution was then applied to the sky subtracted science frame using gstransform. Extraction of the spectra was performed with gsextract using all available light in each slit. All slits were cut to the wavelength range 4800-5500 $\AA$ and rebinned to the same dispersion with dispcor.

\section{1. $p P X F$}

To extract velocities (Sect. 3), and velocity dispersions (Sect. 4), from each slit we employed the Interactive Data Language (IDL) penalised PiXel Fitting software (pPXF; Cappellari \& Emsellem 2004; Cappellari 2012). The stellar spectral templates we used were those from the MILES ${ }^{1}$ library (see also Sánchez-Blázquez et al. 2006), chosen because these templates cover the wavelength range we require, as well as having sufficient resolution for our purposes. Furthermore, the MILES library was chosen to emulate the study by S12. For an in-depth discussion of both pPXF and the MILES stellar library, please see S12.

\section{Slit velocities}

Line-of-sight radial velocities were estimated for each slit directly using pPXF. For most slits the signal-to-noise $(\mathrm{S} / \mathrm{N})$ was high enough $(\gtrsim 5)$ to reliably estimate the velocities (sanity checked with fxcor, see Sect. 4). In a few cases, due to CCD imperfections and because some slits were too short to provide sufficient signal at a given radius, the uncertainty in the slit velocity was determined to be too large for the velocity estimate to be useful. Furthermore, the four slits with the largest radii,

http://miles.iac.es/ 


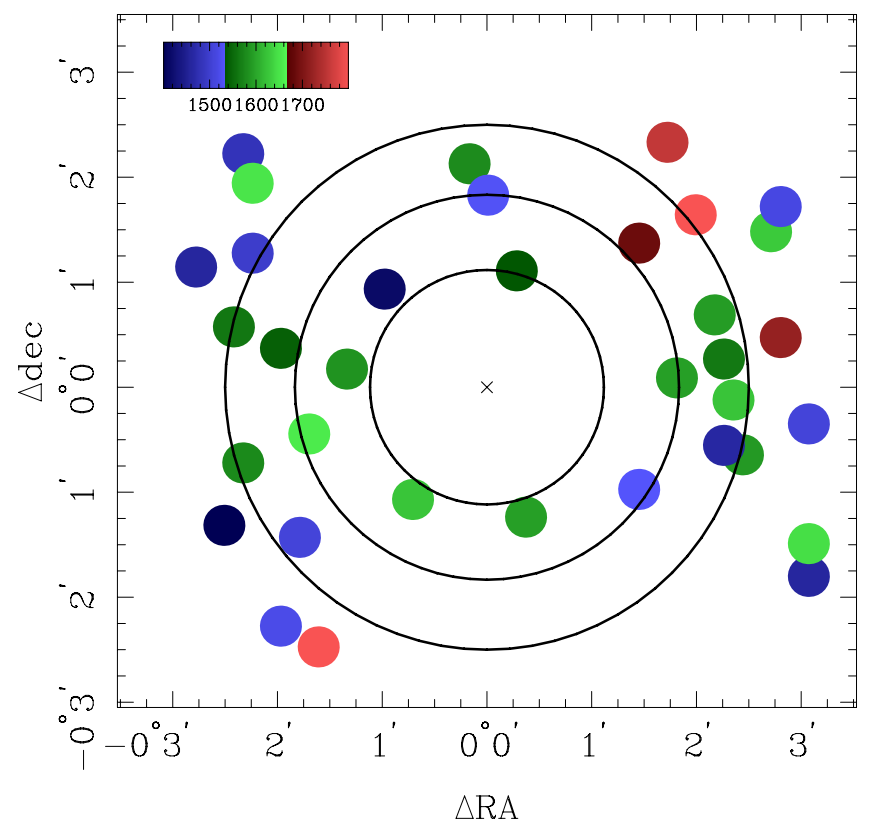

Fig. 2. Velocities of the 36 individual slits with good velocity estimates. The individual spectra are plotted as large coloured points with colours representing their velocities. The legend at the top left is given is $\mathrm{km} \mathrm{s}^{-1}$. The orientation has been chosen to correspond to that of Fig. 1, so that north is down and east is to the left. Large circles delineate the bin edges used in Fig. 3 at 67", 110" and 150".

those with $R>195^{\prime \prime}$, had insufficient $\mathrm{S} / \mathrm{N}$ to provide realistic velocity estimates. These slits were removed, and only those with uncertainties less than $100 \mathrm{~km} \mathrm{~s}^{-1}$ have been included, leaving 36 slits with reliable velocity estimates. The velocities extracted from each individual spectrum are represented in Fig. 2 and listed in Table 1.

\subsection{Rotation}

Using long slit spectra, the rotation of NGC 7507 was first reported by Franx et al. (1989), and later corroborated and extended to larger radii by $\mathrm{S} 12$. The latter measured the rotation of NGC 7507 along the "minor axis" (stated as lying on the line with a position angle of $0^{\circ}$ ) to have a peak amplitude of $\sim 50 \mathrm{~km} \mathrm{~s}^{-1}$ at $R \lesssim 50^{\prime \prime}$, dropping to somewhere between $0-50 \mathrm{~km} \mathrm{~s}^{-1}$, due to large uncertainties, at $60^{\prime \prime} \lesssim R \lesssim 80^{\prime \prime}$.

Employing a slit mask, with slits over all position angles (PAs), allows us to measure the rotation over all PAs, unlike the previous long slit studies. To measure the rotation, we used a common method (see e.g. Lane et al. 2009, and references therein) which involves dividing the slit mask in half at a given PA and calculating difference between the average velocities in the two halves. This was performed over PAs in steps of $25^{\circ}$. Three bins were used, namely slits with $67^{\prime \prime}<R<110^{\prime \prime}$, those with $110^{\prime \prime}<R<150^{\prime \prime}$ and those with $150^{\prime \prime}<R<195^{\prime \prime}$. This binning was chosen so that the bins had similar radial widths and a similar number of slits in each bin. Figure 3 shows the velocities for each step in PA in each individual bin, as well as the overall velocities for each step in PA measured with all the slits at all radii. The best fit sine curve has been overplotted on the resulting rotation curves. Note that the $25^{\circ}$ spacing of the steps in PA is arbitrary and was chosen simply to populate the figure with a sufficiently large number of data points for visual clarity. Taking larger steps in PA simply reduces the number of data
Table 1. Velocity estimates for each slit, as measured by pPXF, listed by increasing slit radius.

\begin{tabular}{|c|c|c|c|}
\hline Slit ID & $R\left({ }^{\prime \prime}\right)$ & $v_{\mathrm{r}}\left(\mathrm{km} \mathrm{s}^{-1}\right)$ & $\delta v_{\mathrm{r}}\left(\mathrm{km} \mathrm{s}^{-1}\right)$ \\
\hline 157 & 67 & 1539 & 9 \\
\hline 181 & 72 & 1586 & 11 \\
\hline 38 & 75 & 1624 & 28 \\
\hline 177 & 76 & 1419 & 17 \\
\hline 150 & 78 & 1594 & 18 \\
\hline 48 & 94 & 1653 & 18 \\
\hline 62 & 95 & 1594 & 16 \\
\hline 178 & 96 & 1535 & 38 \\
\hline 83 & 106 & 1544 & 50 \\
\hline 168 & 109 & 1529 & 31 \\
\hline 175 & 111 & 1685 & 20 \\
\hline 113 & 116 & 1572 & 22 \\
\hline 67 & 120 & 1564 & 25 \\
\hline 111 & 121 & 1591 & 75 \\
\hline 1 & 124 & 1464 & 26 \\
\hline 138 & 124 & 1621 & 33 \\
\hline 176 & 127 & 1580 & 18 \\
\hline 144 & 128 & 1508 & 31 \\
\hline 33 & 131 & 1577 & 28 \\
\hline 45 & 132 & 1562 & 23 \\
\hline 182 & 134 & 1591 & 21 \\
\hline 15 & 141 & 1496 & 30 \\
\hline 27 & 143 & 1889 & 65 \\
\hline 180 & 150 & 1719 & 37 \\
\hline 19 & 155 & 1257 & 76 \\
\hline 50 & 162 & 1463 & 27 \\
\hline 126 & 163 & 1507 & 42 \\
\hline 120 & 166 & 1650 & 21 \\
\hline 163 & 166 & 1753 & 52 \\
\hline 155 & 167 & 1629 & 26 \\
\hline 78 & 172 & 1844 & 82 \\
\hline 52 & 172 & 1518 & 58 \\
\hline 95 & 179 & 1515 & 89 \\
\hline 103 & 181 & 1485 & 23 \\
\hline 82 & 185 & 1645 & 34 \\
\hline 109 & 195 & 1461 & 39 \\
\hline
\end{tabular}

Notes. Columns are: ID of the slit, radius from the galactic centre, line of sight radial velocity and the uncertainty in the radial velocity measurement.

points and does not affect the results in any way. As can be seen in Fig. 3, for those PAs where there are no slits the velocity of those PAs have not been measured.

Serendipitously, the chosen binning also highlights boundaries at which the rotation of the galactic halo appears to change direction. From Fig. 3, it is clear that the projected rotational axis of the galactic halo for radii $67^{\prime \prime}<R<110^{\prime \prime}$ lies approximately along the line PA $=0^{\circ}$, in perfect agreement with the studies by Franx et al. (1989) and S12. While it could be argued that a net rotation of zero may be consistent with the data, within the uncertainties, the best fit amplitude of rotation for this inner bin is $\sim 22 \pm 8 \mathrm{~km} \mathrm{~s}^{-1}$, in agreement with the value given by $\mathrm{S} 12$ at their largest radius of $\sim 80^{\prime \prime}$. For the intermediate bin, $110^{\prime \prime}<R<150^{\prime \prime}$, the projected rotation axis lies approximately along the line PA $=120^{\circ}$. It is clear that the direction of rotation at these intermediate radii has reversed compared with the inner bin, and has a rotational velocity of $\sim 55 \pm 7 \mathrm{~km} \mathrm{~s}^{-1}$. For the outer bin, $150^{\prime \prime}<R<195^{\prime \prime}$, the projected axis of rotation is close to the line $\mathrm{PA}=100^{\circ}$ with the rotation in approximately the same direction as the intermediate bin, and a rotational velocity of $\sim 70 \pm 7$. The overall rotation, measured over all radii, has a projected axis of rotation lying approximately 


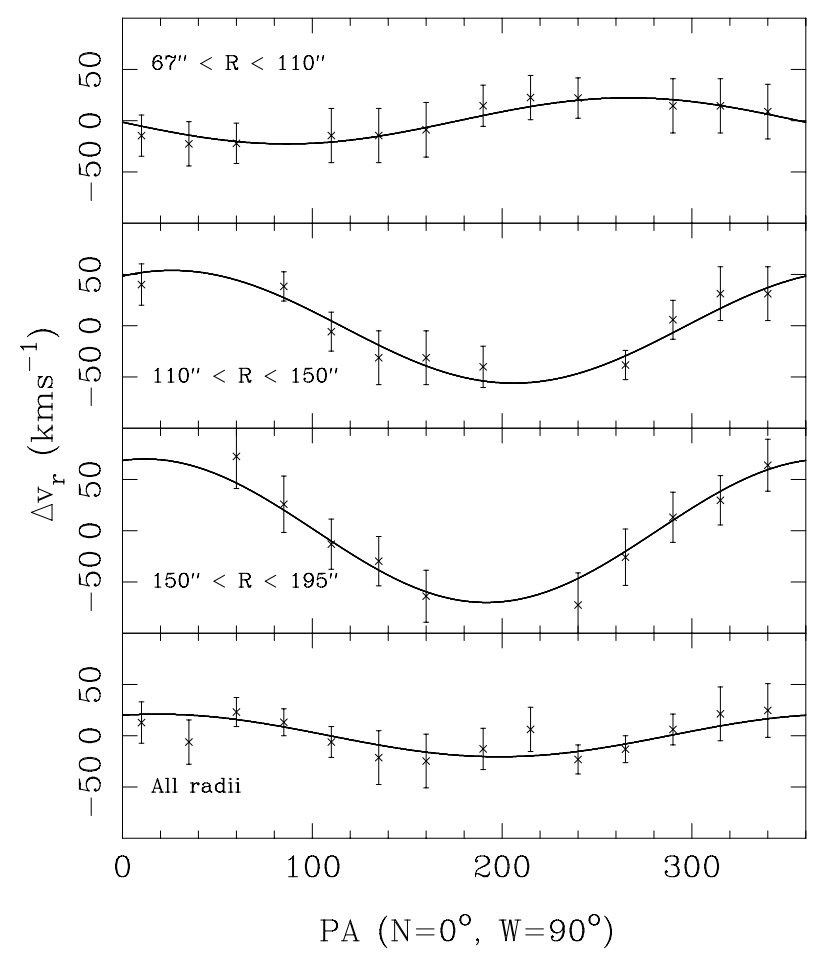

Fig. 3. Rotational velocity versus position angle of the halo of NGC 7507 in three radial bins, shown in each panel, as per the methodology described in the text. The error bars represent the RMS errors for each PA. The best fit sine curve is overplotted. Note that the $\Delta v_{\mathrm{r}}$ values calculated by the method described in the text are twice the actual rotational velocity. We have taken this into account so that the values shown here represent the actual rotational velocity at each PA.

along the line, $\mathrm{PA}=80^{\circ}$, similar to that of the outer halo. At first glance this may seem to indicate that the outer halo contributes most of the rotational velocity to the galaxy, however, because the intermediate bin has a very similar rotational axis to the outer bin, and there are far fewer slits in the inner bin compared to the two outer bins combined, it is clear that this is only a statistical effect.

To ensure that the binning was not a contributing factor to the rotation measurements we rebinned the slits at various different radii, making sure that no bin ever contained fewer than ten slits. Small shifts in the projected axes and amplitude of rotation were found, however, such shifts are to be expected, especially for the two inner bins, where the direction of rotation is reversed. These small differences, and the close agreement at small radii between our results and those of previous studies, indicate that the binning is not affecting our results, and, furthermore, that our estimated rotational amplitudes and axes of rotation are also reliable at large radii. See Sect. 6 for further discussion.

\section{Velocity dispersion}

It was necessary to stack spectra to achieve sufficiently high $\mathrm{S} / \mathrm{N}$ $(\gtrsim 10)$ to allow for accurate velocity dispersion estimates, especially at large radii where there is less galaxy light in each slit. For the stacking, we shifted the newly added spectra in steps of $0.1 \AA$ to locate the shift required to minimise the velocity dispersion. In this way we ensured that the line widths in the stacked spectra were not artificially increased due to velocity shifts in the spectral lines caused by rotation, which would have led to artificial line broadening, and hence an artificial increase
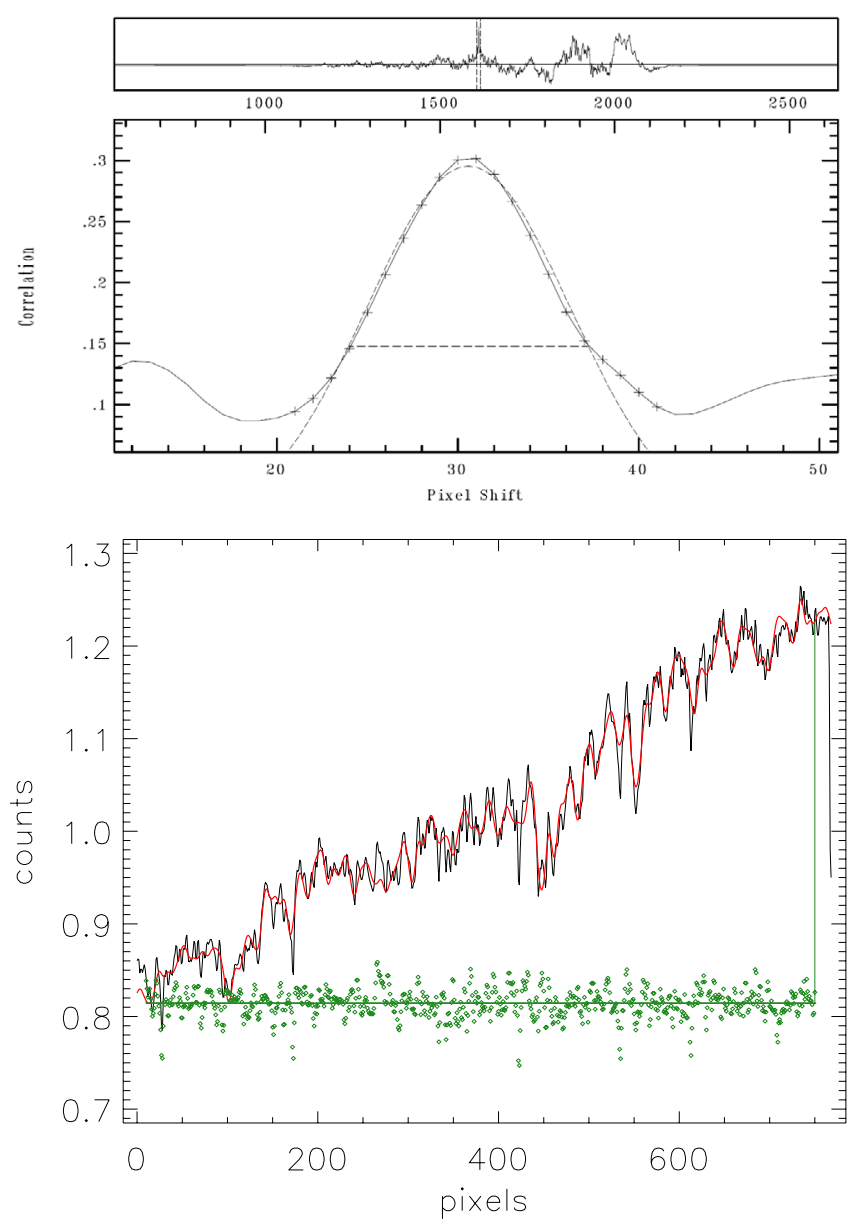

Fig. 4. Combined spectrum from slits with IDs 181,38,177 and 150, $72^{\prime \prime}<R<78^{\prime \prime}$. Top panel: using fxcor in IRAF, the radial velocity for the combined spectrum estimated via cross correlation is $1579 \pm 96 \mathrm{~km} \mathrm{~s}^{-1}$. Bottom panel: the best fit from pPXF (solid red line) to the combined spectrum (solid black line) using 13 templates from the MILES library, gives a radial velocity of $1575 \pm 6 \mathrm{~km} \mathrm{~s}^{-1}$. The residuals from the fit are shown as green points. Both estimates are consistent with the known values for NGC 7507, and are within the uncertainties of each other.

in the velocity dispersion. To test the veracity of the velocity dispersion minima, we checked each shifted/stacked spectrum with the IRAF package $f$ xcor to ensure that the estimated velocity given by $f x c o r$ was the same, within the uncertainties, as that given by pPXF, and also that the minimum velocity dispersion given by pPXF relates to the minimum $F W H M$ of the cross-correlation function given by fxcor (see Fig. 4). The maximum shift for any of the spectra, as compared to the original spectrum, was $2.5 \AA$. Note that $2.5 \AA$ corresponds to a velocity of $\sim 145 \mathrm{~km} \mathrm{~s}^{-1}$ at $5200 \AA$, approximately the central wavelength of our spectra, which is clearly larger than the rotational velocity. However, the mean shift for all the stacked spectra was $\sim 0.08 \AA$, corresponding to a mean velocity of $\sim 4.5 \mathrm{~km} \mathrm{~s}^{-1}$ at $5200 \AA$, which is compatible with zero, given the uncertainties, as would be expected under the assumption of solid-body rotation.

To produce the final stacked spectra for analysis we used a "rolling" stacking procedure as follows. The first, innermost, spectrum was analysed by itself, as it had sufficient $\mathrm{S} / \mathrm{N}$ for an accurate estimate of the velocity dispersion. Then the spectrum from the slit with the second smallest radius was analysed to check whether it had sufficient signal. If not, the spectrum 

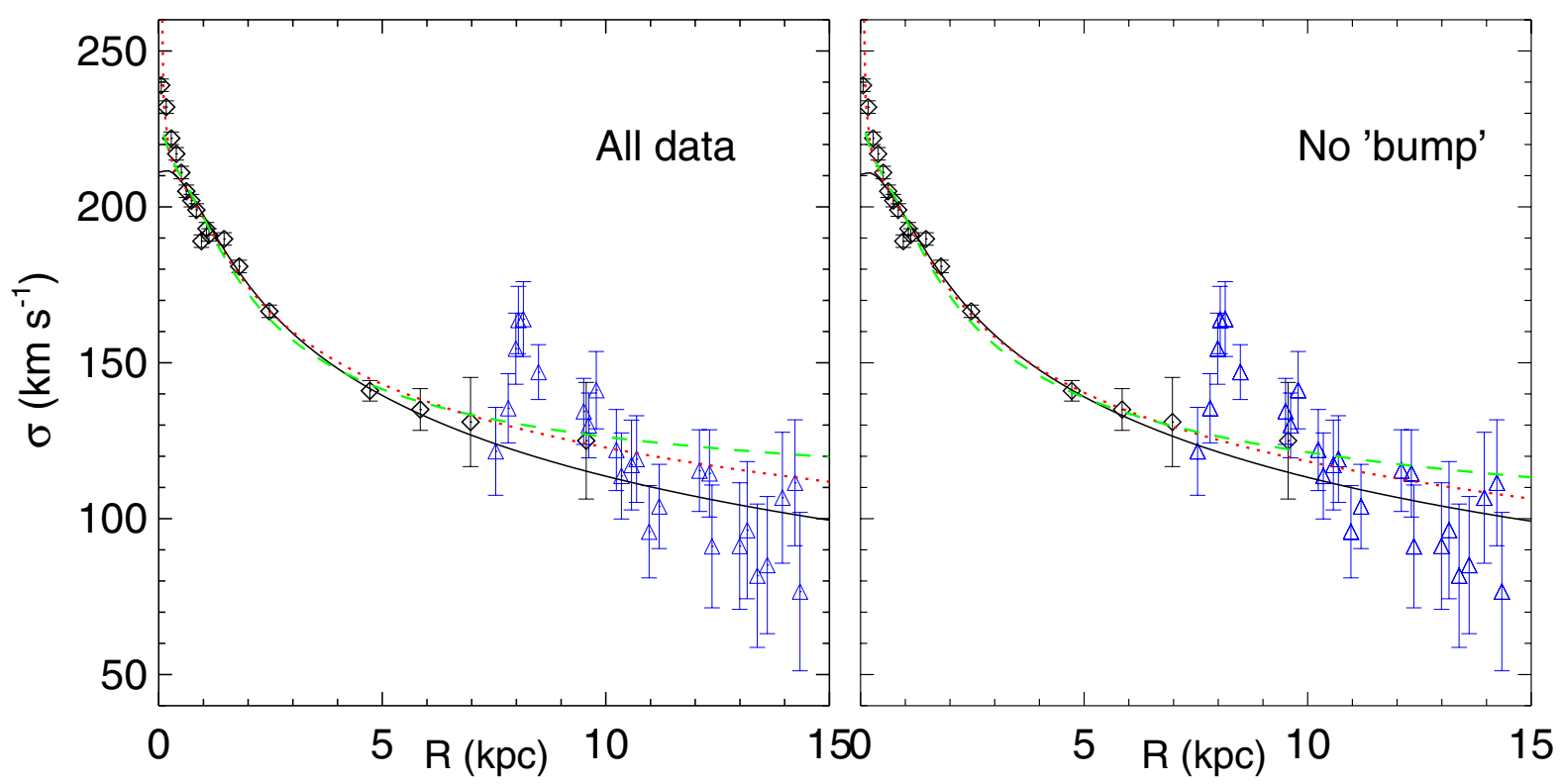

Fig. 5. Dynamical models. Black open diamonds are the measurements from S12, while blue triangles are the velocity dispersions from the current work. Left panel: models which consider all the velocity dispersion points. The solid black curve is the stars-only model. The red dotted curve is the best-fitting model with $\beta=0.3$ and NFW halo with $c=10$. The green dashed curve is the model under the anisotropy by Mamon \& Łokas (2005) allowing the maximum amount of dark mater. Right panel: same as in left panel, but excluding the velocity dispersion between $70^{\prime \prime}<R<80^{\prime \prime}$ when performing the fits.

from the slit with the next smallest radius was added (stacked). This process continued until there was sufficient signal to extract a reliable velocity dispersion measurement. Once the measurement was performed, the spectrum with the next smallest radius was added and the spectrum with the smallest radii from the previously stacked spectrum was removed, this new spectrum was then checked for sufficient signal, and so on. This procedure produces stacked spectra that are binned by increasing radius, with varying bin widths, and with overlapping bin edges, unlike more traditional binning. This technique was necessary as the available number of spectra was small. Producing binned spectra in a more traditional way, where spectra are binned without overlapping bin edges, would have produced velocity dispersion estimates at only a few radii and removed information from our analysis. Using rolling bins is a common way to overcome this problem (e.g. Blom et al. 2012; Gerke et al. 2013; Foster et al. 2013). It should be noted that the $\mathrm{S} / \mathrm{N}$ in the innermost few slits was low, due to short slit lengths, and it was difficult to extract sufficient signal at these radii, except in the innermost slit. It was, therefore, useful to include the first slit, which had much higher $\mathrm{S} / \mathrm{N}$, to the innermost four stacked spectra. To stack the spectra we average combined them using the scombine task within IRAF. At a radius of $\sim 134^{\prime \prime}$ the uncertainties in the velocity dispersions, as well as the scatter between individual measurements, began to overwhelm the measurements themselves. Therefore, we decided to restrict our velocity dispersion estimates to $R \lesssim 134^{\prime \prime}$. The velocity dispersion estimates are shown in Table 2 .

The velocity dispersion profile is shown in Fig. 5, where the radii of the individual data points reflect the mean radius of the stacked slits employed to determine the velocity dispersion. At radii where the data can be directly compared, our velocity dispersion estimates are completely consistent with those by $\mathrm{S} 12$, who used long slit spectroscopy to determine velocity dispersions.

Interestingly, it is clear from Fig. 5 that there is a sharp increase in the velocity dispersion at $67^{\prime \prime} \lesssim R \lesssim 70^{\prime \prime}$.
Table 2. Velocity dispersions produced using the rolling stacking technique, as described in the text, represented as the open squares in Fig. 5.

\begin{tabular}{ccc}
\hline \hline$R\left({ }^{\prime \prime}\right)$ & $\sigma_{v}\left(\mathrm{~km} \mathrm{~s}^{-1}\right)$ & $\delta \sigma_{v}\left(\mathrm{~km} \mathrm{~s}^{-1}\right)$ \\
\hline 67 & 121.6 & 14.1 \\
$67-72$ & 135.4 & 11.1 \\
$67-75$ & 154.5 & 11.4 \\
$67-76$ & 163.7 & 10.8 \\
$67-78$ & 164.0 & 12.0 \\
$72-79$ & 147.0 & 8.8 \\
$75-94$ & 134.4 & 10.6 \\
$76-95$ & 129.9 & 10.4 \\
$78-96$ & 141.2 & 12.4 \\
$78-104$ & 122.0 & 13.0 \\
$78-106$ & 113.7 & 13.8 \\
$79-109$ & 117.2 & 14.4 \\
$79-111$ & 119.1 & 13.9 \\
$79-116$ & 95.8 & 14.8 \\
$79-120$ & 103.9 & 13.5 \\
$94-121$ & 115.4 & 13.1 \\
$95-124$ & 114.5 & 14.0 \\
$96-124$ & 91.1 & 19.7 \\
$104-127$ & 91.2 & 20.3 \\
$106-128$ & 96.3 & 22.0 \\
$109-129$ & 81.7 & 23.0 \\
$111-131$ & 85.1 & 22.0 \\
$116-132$ & 106.7 & 21.0 \\
$120-133$ & 111.5 & 20.2 \\
$121-134$ & 76.6 & 25.4 \\
\hline
\end{tabular}

Notes. The columns are: 1 - the radii, in arcseconds, of the slits combined to produce the final stacked spectra, 2 - the velocity dispersion of the final stacked spectra, and 3 - the uncertainty on the velocity dispersion estimate.

In the context of a spherically symmetric model, this increase is unphysical. It has been shown, however, that stellar remnants from mergers can be very long lived in dwarf spheroidal galaxies (at least up to $10 \mathrm{Gyr}$ ) and, despite being difficult to 
A\&A 574, A93 (2015)

Table 3. Comparison of different best fitting dynamical models including NFW haloes.

\begin{tabular}{|c|c|c|c|c|c|c|c|}
\hline $\begin{array}{l}M / L_{\text {stars }} \\
\left(M_{\odot} / L_{\odot}\right)\end{array}$ & $\beta$ & $c$ & $\begin{array}{c}\rho_{\mathrm{s}} \\
\left(10^{-3} \times M_{\odot} \mathrm{pc}^{-3}\right)\end{array}$ & $\begin{array}{c}r_{\mathrm{s}} \\
(\mathrm{kpc})\end{array}$ & $\begin{array}{l}M_{200}^{\mathrm{DM}} \\
\left(M_{\odot}\right)\end{array}$ & $\chi^{2}$ & $\chi^{2} /$ d.o.f. \\
\hline \multicolumn{8}{|c|}{ all data } \\
\hline 3.15 & $0^{*}$ & - & - & - & - & 111.43 & 2.86 \\
\hline 3.156 & -0.043 & - & - & - & - & 111.19 & 2.85 \\
\hline 3.08 & $0 *$ & $5^{*}$ & $1.18249 *$ & 23.5 & $1.85 \times 10^{11}$ & 100.4 & 2.71 \\
\hline 3.06 & $0^{*}$ & $10 *$ & $6.08990 *$ & 9.0 & $8.31 \times 10^{10}$ & 103.1 & 2.79 \\
\hline 2.96 & $0.3^{*}$ & $5^{*}$ & $1.18249 *$ & 34.8 & $6.00 \times 10^{11}$ & 89.6 & 2.39 \\
\hline 2.91 & $0.3^{*}$ & $10 *$ & $6.08990 *$ & 13.3 & $2.68 \times 10^{11}$ & 88.1 & 2.38 \\
\hline 2.78 & ME* & $5^{*}$ & $1.18249 *$ & 42.8 & $1.12 \times 10^{12}$ & 97.3 & 2.56 \\
\hline 2.78 & MŁ* & 10 & $6.08990 *$ & 16.0 & $4.67 \times 10^{11}$ & 92.8 & 2.44 \\
\hline \multicolumn{8}{|c|}{ No "bump" } \\
\hline 3.13 & $0^{*}$ & - & - & - & - & 65.08 & 1.86 \\
\hline 3.12 & 0.072 & - & - & - & - & 64.22 & 1.88 \\
\hline 3.11 & $0 *$ & $5^{*}$ & $1.18249 *$ & 12.45 & $2.75 \times 10^{10}$ & 64.46 & 1.89 \\
\hline 3.13 & $0 *$ & $10 *$ & $6.08990 *$ & 1.875 & $7.51 \times 10^{8}$ & 65.21 & 1.91 \\
\hline 2.98 & $0.3^{*}$ & $5^{*}$ & $1.18249 *$ & 27.7 & $3.03 \times 10^{11}$ & 56.5 & 1.66 \\
\hline 2.94 & $0.3^{*}$ & $10 *$ & $6.08990 *$ & 11.0 & $1.52 \times 10^{11}$ & 55.5 & 1.63 \\
\hline 2.80 & MŁ* & $5^{*}$ & $1.18249 *$ & 36.5 & $6.93 \times 10^{11}$ & 67.5 & 1.98 \\
\hline 2.74 & MŁ* & 10 & $6.08990 *$ & 14.2 & $3.27 \times 10^{11}$ & 62.6 & 1.84 \\
\hline
\end{tabular}

Notes. Quantities with an asterisk mean they have kept fixed during the minimisation process. The "no bump" panel refers to the quantities derived when the velocity dispersion values between $70^{\prime \prime}$ and 80" are removed. MŁ refers to the Mamon \& Łokas (2005) anisotropy profile of Eq. (2).

observe photometrically, can lead to increased velocity dispersions (Assmann et al. 2013a,b). There is a very slight red colour excess about $10^{\prime \prime}-15^{\prime \prime}$ southwest of the galactic centre (S12; Caso et al. 2013), which may be explained by cold dust (also see Temi et al. 2004). Apart from this, no obvious signatures of past mergers are visible in photometric data.

Recent numerical merger simulations by Schauer et al. (2014) were performed to try to explain what they call the " $\sigma$ bump". This bump is a flattening of the line of sight velocity dispersion profile at radii between $\sim 1-3 R_{\mathrm{e}}$ (similar to what is seen, for example, in NGCs 821, 3115 and 4278, by: Coccato et al. 2009; Arnold et al. 2011; Pota et al. 2013). The bump is long-lived - at the end of their longest simulation of 9 Gyr the bump was effectively unchanged. However, other kinematic remnants, such as shells, dissipate over much shorter time scales and are no longer visible after $\sim 2$ Gyr. Note that the authors simulate mergers between a spiral galaxy and an elliptical galaxy, and between two spiral galaxies. The bump is more pronounced in the spiral-spiral mergers, although it is still visible in spiral-elliptical mergers. It should also be noted that the shape of the bump, at least in the simulations, does not change with the inclination of the projection, even though the overall velocity dispersion does decrease with increasing projection angle. Furthermore, sophisticated $N$-body/hydrodynamic merger simulations by Ji et al. (2014) show features from major mergers last 30\% longer in isolated mergers compared with mergers in cluster environments.

In the simulations by Schauer et al. (2014), this "bump" is seen as a velocity dispersion that is fairly constant with radius. However, in NGCs 821, 3115 and 4278, and now in NGC 7507, there is an increase in the velocity dispersion, rather than a flattening. In the case of NGC 821 (Coccato et al. 2009; Pota et al. 2013), the increase in velocity dispersion is comparable with what we observe in NGC $7507\left(\sim 50 \mathrm{~km} \mathrm{~s}^{-1}\right)$. Even though the simulations are not a perfect match to the data in any of these cases, they lend credibility to the idea of merger activity being the explanation for this deviation from the expectations of spherical symmetry.

\section{Dynamical models}

We now use the light model that we produced in S12 to investigate, by simple dynamical modelling, whether a dark halo is present and what stellar $M / L$ values are required. As in $\mathrm{S} 12$, we performed spherical Jeans modelling. Since NGC 7507 is an E0 (e.g. de Souza et al. 2004) with a small rotational velocity (Sect. 3.1), the assumption of sphericity is reasonable, as a first approximation (see also S12). A compilation of the relevant formulae can be found in Mamon \& Łokas (2005) and Schuberth et al. (2010), and a detailed discussion of the process can be found in S12 (and references therein).

Our modelling is based on the spherical Jeans equation, which is only valid for non-rotating systems. Its solutions, however, are considered to be good approximations for elliptical galaxies, in part because anisotropies in the inner regions are small, and it has the advantage of simplicity. Furthermore, results from more sophisticated dynamical analyses do not differ greatly from our approach. The bulk of the our uncertainties derive from the uncertainty in distance and the data quality, rather than the modelling. We multiply the deprojected light model with a stellar $M / L$ value and calculate line-of-sight velocity dispersions after adding, or not adding, a dark halo, and with certain assumptions concerning the anisotropy.

We repeat the analysis of $\mathrm{S} 12$, but restricted to a subset of the most interesting models, without including, for example, the case of logarithmic haloes. Table 3 gives the results for the selected models. As in S12, the models which minimise $\chi^{2}$ are considered as the best-fitting ones. The inner $3^{\prime \prime}(\sim 330 \mathrm{pc})$ are not considered in the minimisation given the poor description of the (saturated) light profile in that range.

The first, and simplest, model is the one where only stars are considered, under isotropy $(\beta=0)$. This model describes the data very well (solid black curve in the left panel of Fig. 5), implying a stellar $M / L_{R}=3.13$. If anisotropy is left as a free parameter, there is a negligible change from $\beta=0$ to $\beta=-0.043$, without a discernable improvement to the fit. 
The next step is to include a dark matter halo described by a NFW profile (Navarro et al. 1997),

$\rho(r)=\frac{\rho_{\mathrm{s}}}{\left(r / r_{\mathrm{s}}\right)\left(1+r / r_{\mathrm{s}}\right)^{2}}$,

where $\rho_{\mathrm{s}}$ and $r_{\mathrm{s}}$ are the characteristic density and radius, respectively. As in S12, we restrict the analysis to two concentrations $\left(c=r_{200} / r_{\mathrm{s}}\right)$ of 5 and 10 , both under isotropy and with a mild radial anisotropy $(\beta=0.3)$. All the models including a dark halo provide better fits (lower $\chi^{2}$ ) than the stars-only model, although the amount of dark matter is very low, and the difference in the goodness of fit is small. Our most massive halo has a mass of $6 \times 10^{11} M_{\odot}$, although a halo mass of $2.68 \times 10^{11} M_{\odot}$ provides a better fit to the data, and our overall best fit model, which excludes the bump (see below), has a dark halo mass of only $1.5 \times 10^{11} M_{\odot}$ (which corresponds to a dark matter halo concentration, $c_{200}$, of about 6 , based on the cosmology from WMAP data release 3, Macciò et al. 2008). Even if we assume our maximum halo mass of $6 \times 10^{11} M_{\odot}$, this would imply a stellar mass of $\lesssim 2 \times 10^{10} M_{\odot}$ (e.g. Niemi et al. 2010), 10 times lower than the light profile would suggest (S12). If we adopt a stellar mass of $\sim 2 \times 10^{11} M_{\odot}(\mathrm{S} 12)$ then this implies a dark halo mass $\gtrsim 10^{13} M_{\odot}$, a factor of at least 100 greater than described by our best fit model. For clarity, Fig. 5 (left panel) only shows the results for the model with $\beta=0.3$ giving the lowest $\chi^{2}$. All other models are visually very similar.

As discussed in the previous section, the velocity dispersion profile exhibits a small increase, or bump, between $70^{\prime \prime}<R<$ $80^{\prime \prime}$. We consider now the influence of this feature in the previous minimisation. The lower panel of Table 3 includes the same models described above, but this time doing the minimisation excluding the bump region. It is clear that the isotropic NFW models no longer improve the $\chi^{2} /$ d.o.f. when compared to the dark matter free models, although the mildly radial models continue to give the lowest $\chi^{2}$ values.

It is possible that the anisotropy profile of elliptical galaxies is not constant, but rather increasingly radial at larger radii (e.g. Dekel et al. 2005; Hansen et al. 2006). For collisionless mergers, Hansen et al. (2006) suggest a universal relation between the anisotropy parameter $\beta$ of the Jeans equation and the logarithmic slope of the density profile, $\alpha$, of the form $\beta(\alpha)=$ $1-1.15(1+\alpha / 6)$. For our density profile this means a quick rise up to $\beta=0.4$ at $\sim 2 \mathrm{kpc}$, followed by a smooth increase to $\beta=0.5$ at $\sim 15 \mathrm{kpc}$. This behaviour can be well approximated by the anisotropy profile of Mamon \& Łokas (2005),

$\beta_{\mathrm{MŁ}}(r)=\beta_{0} \frac{r}{r+r_{\mathrm{a}}}$,

using $\beta_{0}=0.5$ and $r_{\mathrm{a}}=500 \mathrm{pc}$. The dark matter haloes which minimise the $\chi^{2}$ using this form of anisotropy can also be seen in Table 3 for both the complete dataset and the one without the bump region. As expected from the mass-anisotropy degeneracy, highly radial orbits allow for higher mass haloes, up to $1 \times 10^{12} M_{\odot}$ in the case of $c=5$, although giving systematically poorer fits compared to the $\beta=0.3$ case. In the case of $c=5$ with the "no bump" velocity dispersions, the fit is even worse than the models without dark matter.

It is also worth considering whether MOND (Milgrom 1983) can reproduce better the observed velocity dispersion profile. In $\mathrm{S} 12, \mathrm{MOND}$ overpredicted the velocity dispersion at large radii, even when considering the most favourable cases including a large radial anisotropy. Those results were later questioned by Famaey \& McGaugh (2012; see their Fig. 37) who quoted unpublished work by Sanders, which we cannot assess.

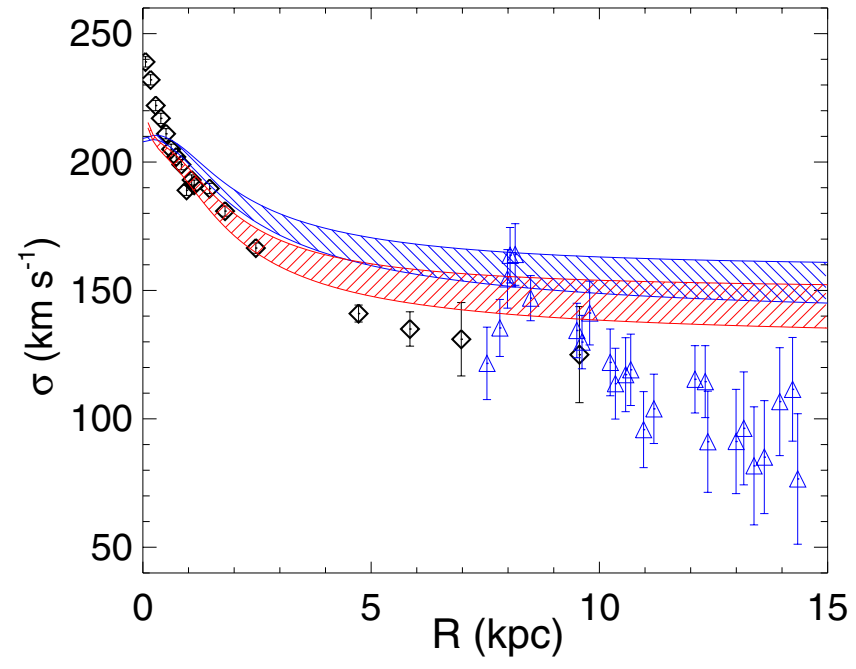

Fig. 6. MOND models. As in Fig. 5, black diamonds are the measurements from $\mathrm{S} 12$, while blue triangles are the velocity dispersions from the current work. The blue hatched area covers isotropic models with the range of $a_{0}$ values discussed in the text, while the red area represents the same range for $a_{0}$ but under the Mamon \& Łokas (2005) anisotropy profile.

The MOND velocity dispersions are obtained by first calculating the MOND associated circular velocity from the Newtonian circular velocity using:

$v_{\mathrm{M}}=\sqrt{v_{N}^{2}(r) / 2+\sqrt{v_{N}^{4}(r) / 4+v_{N}^{2}(r) a_{0} r}}$

which assumes the simple interpolation formula of Famaey \& Binney (2005) and where $a_{0}=1.35_{-0.42}^{+0.28} \times 10^{-8} \mathrm{~cm} \mathrm{~s}^{-2}$ (Famaey et al. 2007). The mass associated with this MONDian circular velocity is then introduced in the same set of formulae quoted above (see also Richtler et al. 2011b). Figure 6 shows the new MOND models including the extended measurements presented in this paper. As in S12 we calculate MOND models encompassing the range of accepted $a_{0}$ values by Famaey et al. (2007). The hatched blue area indicates the MOND predictions under isotropy $\left(M / L_{R}=3.0\right)$, while the red area uses the anisotropy profile from Eq. (2) $\left(M / L_{R}=2.5\right)$. Regardless of the assumed anisotropy profile, or the adopted value of $a_{0}$, MOND systematically fails to reproduce the outer velocity dispersion profile.

Another issue to explore are variations in $M / L$. However, there is no indication from the colour profile that the stellar $M / L$ should vary (S12). The age of $\sim 4 \pm 1$ Gyr by Li et al. (2007) indicates $M / L_{R} \sim 2.1$ (Bressan et al. 2012), however, the colours of the galaxy indicate an age closer to $8 \mathrm{Gyr}$ and $M / L_{R} \sim 3.9$ (Fig. 7), and a $M / L_{R}$ as low as 2.1 does not match our measured dynamics. Note that in Fig. 7 we have used a Chabrier (2003) IMF, which is fairly bottom-light, in contrast with recent considerations for some elliptical galaxies (e.g. van Dokkum \& Conroy 2010; Dutton et al. 2012; Tortora et al. 2013). However, all such recent studies have focussed on elliptical galaxies in dense environments. While it seems that massive elliptical galaxies can have more bottom heavy IMFs in such environments, it is not at all clear that isolated elliptical galaxies also have bottom heavy IMFs. The age of the galaxy (as young as $\sim 4 \mathrm{Gyr}, \mathrm{Li}$ et al. 2007) may accommodate a lower $M / L$ through younger populations. Furthermore, the maximal $M / L$ is that of the "maximal spheroid", with no dark matter and isotropy. Any dark matter or radial anisotropy boosts the central velocity dispersion and requires a lower $M / L$ as seen in Table 3 . 


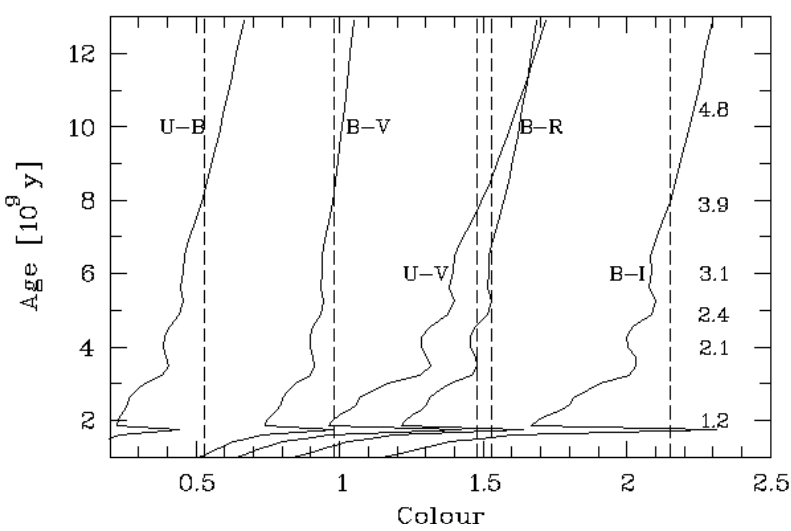

Fig. 7. Age versus colour. The solid lines represent the models by Marigo et al. (2008) and the vertical dashed lines are the colours by Prugniel \& Heraudeau (1998) using a 30" aperture. On the right the $M / L_{R}$ values for a given age (Chabrier $2003 \mathrm{IMF}-$ using $M_{\odot}=+4.42$ ) are shown. The $(B-R)$ colour is not really age discriminative, however, an age of $\sim 8 \mathrm{Gyr}$ and $M / L_{R} \sim 4$ is consistent with all other colours (cf. an age of $\sim 4 \pm 1 \mathrm{Gyr}$ by $\mathrm{Li}$ et al. 2007, based on $[B-V]$ and $[B-K]$ colours and the models by Bruzual \& Charlot 2003). Note that a $M / L_{R}$ of 3.1 is well described by our dynamics, but 2.8 is still possible (Table 3). A $M / L_{R}$ as small as $\sim 2$, to accommodate the age estimate by Li et al. (2007), does not match our dynamics.

As a final note, it is possible that the galaxy is significantly flattened along the line of sight. Two scenarios may result in this flattening, namely that NGC 7507 is, in fact, an S0 galaxy seen face on, or that it is rapidly rotating with the rotational axis close to the plane of the sky (recall that NGC 7507 is circular along the line of sight). Either of these possibilities would lower the velocity dispersion at large radii, which may mimic our measured dynamics. The assumption of flattening is, however, problematic due to the measured rotation of the galaxy. See Sect. 6 for further discussion.

\section{Discussion and conclusions}

Using the penalised PiXel Fitting (pPXF) software by Cappellari \& Emsellem (2004), we have analysed 36 GMOS slit mask spectra of the galaxy light of NGC 7507. This allowed us to calculate a velocity field out to $\sim 195^{\prime \prime}(\sim 22 \mathrm{kpc})$, and a velocity dispersion profile out to $\sim 130^{\prime \prime}(\sim 14.5 \mathrm{kpc})$. This corresponds to between $\sim 2.6-6.3 R_{\mathrm{e}}$ for the velocity field and $\sim 1.7-4.2 R_{\mathrm{e}}$ for the velocity dispersion profile.

Even though the number of slits we used for measuring the rotation of NGC 7507 is small, our measured rotation is completely consistent with other studies. We show evidence for the rotational direction changing in the outer halo, at $R \sim 100^{\prime \prime}$, consistent with results by S12. A change in the direction of halo rotation at large radii has been observed in other galaxies, and there are indications that the outer halo of the Milky Way exhibits the same phenomenon (Carollo et al. 2007; see also Deason et al. 2011). It seems plausible, as suggested by both Carollo et al. (2007) and Deason et al. (2011) in the case of the Milky Way, that the counterrotating outer halo of NGC 7507 is the extant dynamical remnant of an accreted satellite.

While our velocity dispersion estimates agree very well with those by $\mathrm{S} 12$, at radii where our results directly overlap, we have uncovered a peak in the velocity dispersion at $R \sim$ $70^{\prime \prime}, 0.9-2.3 R_{\mathrm{e}}$, undetected by those authors. The most likely explanation for this is also the dynamical remnant of an accretion event. The slits stacked to estimate the velocity dispersion of the four bins exhibiting the increased velocity dispersion have PAs $150^{\circ}-200^{\circ}$ apart, i.e. close to opposite sides of the galaxy, as would be expected by a streaming or bulk motion of stars. Coherent streams can be difficult to detect photometrically but can lead to increases in velocity dispersions and can last for at least 10 Gyr (Assmann et al. 2013a,b). Furthermore, simulations of galaxy mergers by Schauer et al. (2014) reveal a "bump" in the velocity dispersion between $1-3 R_{\mathrm{e}}$ that can be detected at least $9 \mathrm{Gyr}$ after the merger event whereas other merger signatures, such as shells, disappear after $\sim 2$ Gyr. This is further indicative that the increase in velocity dispersion is due to a merger remnant and, since no shells or other signatures of a merger are detected, that the merger occurred more than $\sim 2$ Gyr ago. While this dispersion increase may have another, as yet unknown, origin, we are working under the assumption that it is the signature of a merger remnant. We cannot conclude anything definitive regarding the nature of the interaction. However, from simulations by Schauer et al. (2014) it appears that major mergers impart more of a bump than minor mergers, as do spiral-spiral interactions, hence, a major spiral-spiral merger could be the culprit. It is possible, therefore, that NGCs 821, 3115 and 4278, and 7507 have all formed from spiral-spiral major mergers. We cannot confirm this possibility and further study is required to resolve the evolutionary histories of these galaxies.

Using Jeans and MONDian modelling we have produced various dynamical models in an attempt to determine whether dark matter can be accommodated within our data and the age of NGC 7507, or if MOND can reproduce our measured dynamics. Even with anisotropic models we can only accommodate a small amount dark matter, at least a factor of 100 less than predicted by $\Lambda \mathrm{CDM}$ simulations. These models provide the best fit (lowest $\chi^{2}$ /d.o.f.), although the $\chi^{2} /$ d.o.f. of these models is only marginally lower than that of our dark matter free models. Furthermore, our MONDian models fail to reproduce the measured dynamics at almost all radii. One possibility is that the galaxy is significantly flattened along the line of sight. If NGC 7507 is, in fact, a face-on S0 galaxy, this might also explain the increase in velocity dispersion at $\sim 70^{\prime \prime}$ since simulations have also shown that S0 galaxies can be formed from spiral-spiral mergers (Bekki 1998), and, as discussed, spiralspiral mergers result in a larger $\sigma$ bump than other merger types. It is, however, notoriously difficult to distinguish face-on S0 galaxies from elliptical galaxies (e.g. Tran et al. 2003; Bamford et al. 2009; Weinmann et al. 2009). Rapid rotation may also be the result of a major merger. If NGC 7507 is an S0, or is flattened due to rotation, the change in direction of the inner and outer haloes implies that the inner and outer regions of the "disc" are rotating in different directions, effectively perpendicular to each other (recall that if it is flattened it must be flattened along the line of sight and we must, therefore, be looking at it face-on). We are unaware of any $\mathrm{S} 0$ or rotationally flattened elliptical galaxies where the inner and outer disc regions are rotating with respect to one another in this manner. We are also unaware of any mechanism that may cause this kind of dynamical anomaly.

It should be noted that de Lorenzi et al. (2009) and Napolitano et al. (2009) also found that only very high anisotropy models can accommodate dark matter in the group elliptical galaxies NGC 3379 and NGC 4494 (see also Morganti et al. 2013). Adding to the confusion, Kassin et al. (2006), after decomposing the rotation curves of galaxies into dark and baryonic components, found that, in the case of the spiral galaxy NGC 157, an NFW dark matter halo is inconsistent with the shape of the rotation curve, unless baryons are a significant contributor to the mass within the inner $\sim 5$ scale lengths. 
It appears, then, that dark matter is not necessarily required to explain the dynamics of NGC 7507, nor some other elliptical galaxies in various environments. At the very least the dark haloes of these galaxies, including NGC 7507, are much less massive than expected from $\Lambda \mathrm{CDM}$ simulations. Moreover, in at least one case, dark matter is not required to reproduce the measured rotation curve of a large spiral galaxy. In the context of $\Lambda \mathrm{CDM}$ this is an interesting problem that requires a solution. It is apparent that a systematic study of all galaxies of intermediate luminosities with apparent paucities of dark matter is needed, and we will be addressing this in a forthcoming paper.

Acknowledgements. R.R.L. acknowledges financial support from FONDECYT project No. 3130403. T.R. acknowledges financial support from FONDECYT project No. 1100620, and from the BASAL Centro de Astrofisica y Tecnologias Afines (CATA) PFB-06/2007. T.R. also acknowledges a visitorship at ESO/Garching. We would like to thank Nicola R. Napolitano for his helpful comments and input, which has lead to a more well rounded paper.

\section{References}

Arnold, J. A., Romanowsky, A. J., Brodie, J. P., et al. 2011, ApJ, 736, L26 Assmann, P., Fellhauer, M., Wilkinson, M. I., \& Smith, R. 2013a, MNRAS, 432, 274

Assmann, P., Fellhauer, M., Wilkinson, M. I., Smith, R., \& Blaña, M. 2013b, MNRAS, 435, 2391

Bamford, S. P., Nichol, R. C., Baldry, I. K., et al. 2009, MNRAS, 393, 1324 Bekki, K. 1998, ApJ, 502, L133

Bertin, G., Bertola, F., Buson, L. M., et al. 1994, A\&A, 292, 381

Blom, C., Forbes, D. A., Brodie, J. P., et al. 2012, MNRAS, 426, 1959

Bressan, A., Marigo, P., Girardi, L., et al. 2012, MNRAS, 427, 127

Bruzual, G., \& Charlot, S. 2003, MNRAS, 344, 1000

Cappellari, M. 2012, Astrophysics Source Code Library [ascl:1210.002]

Cappellari, M., \& Emsellem, E. 2004, PASP, 116, 138

Caso, J. P., Richtler, T., Bassino, L. P., et al. 2013, A\&A, 555, A56

Carollo, D., Beers, T. C., Lee, Y. S., et al. 2007, Nature, 450, 1020

Chabrier, G. 2003, PASP, 115, 763

Ciardullo, R., Jacoby, G. H., \& Dejonghe, H. B. 1993, ApJ, 414, 454

Coccato, L., Gerhard, O., Arnaboldi, M., et al. 2009, MNRAS, 394, 1249

Deason, A. J., Belokurov, V., \& Evans, N. W. 2011, MNRAS, 411, 1480

Dekel, A., Stoehr, F., Mamon., G. A., et al. 2005, Nature, 437, 707

de Lorenzi, F., Gerhard, O., Coccato, L., et al. 2009, MNRAS, 395, 76

de Souza, R. E., Gadotti, D. A., \& dos Anjos, S. 2004, ApJS, 153, 411

Douglas, N. G., Napolitano, N. R., Romanowsky, A. J., et al. 2007, ApJ, 664, 257

Dutton, A. A., Mendel, J. T., \& Simard, L. 2012, MNRAS, 422, L33

Famaey, B., \& Binney, J. 2005, MNRAS, 363, 603

Famaey, B., \& McGaugh, S. 2012, Liv. Rev. Relativity, 15, 10

Famaey, B., Gentile, G., Bruneton, J., \& Zhao, H. 2007, Phys. Rev. D, 75, 063002

Foster, C., Proctor, R. N., Forbes, D. A., et al. 2009, MNRAS, 400, 2135
Foster, C., Spitler, L. R., Romanowsky, A. J., et al. 2011, MNRAS, 415, 3393 Foster, C., Arnold, J. A., Forbes, D. A., et al. 2013, MNRAS, 435, 3587

Franx, M., Illingworth, G., \& Heckman, T. 1989, ApJ, 344, 613

Gerke, B. F., Wechsler, R. H., Behroozi, P. S., et al. 2013, ApJS, 208, 1

Hansen, S. H., Moore, B., Zemp, M., \& Stadel, J. 2006, J. Cosmol. Astropart. Phys., 1, 14

Jensen, J. B., Tonry, J. L., Barris, B. J., et al. 2003, ApJ, 583, 712

Ji, I., Peirani, S., \& Yi, S. K. 2014, A\&A, 566, A97

Kassin, S. A., de Jong, R. S., \& Weiner, B. J. 2006, ApJ, 643, 804

Kelson, D. D., Zabludoff, A. I., Williams, K. A., et al. 2002, ApJ, 576, 720

Kronawitter, A., Saglia, R. P., Gerhard, O., \& Bender, R. 2000, A\&AS, 144, 53

Lane, R. R., Kiss, L. L., Lewis, G. F., et al. 2009, MNRAS, 400, 917

Li, Z., Han, Z., \& Zhang, F. 2007, A\&A, 464, 853

Macciò, A. V., Dutton, A. A., \& van den Bosch, F. C. 2008, MNRAS, 391, 1940

Mamon, G. A., \& Łokas, E. L. 2005, MNRAS, 363, 705

Marigo, P., Girardi, L., Bressan, A., et al. 2008, A\&A, 482, 883

Méndez, R. H., Teodorescu, A. M., Kudritzki, R.-P., \& Burkert, A. 2009, ApJ, 691, 228

Milgrom, M. 1983, ApJ, 270, 365

Morganti, L., Gerhard, O., Coccato, L., Martinez-Valpuesta, I., \& Arnaboldi, M. 2013, MNRAS, 431, 3570

Napolitano, N. R., Romanowsky, A. J., Coccato, L., et al. 2009, MNRAS, 393, 329

Napolitano, N. R., Romanowsky, A. J., Capaccioli, M., et al. 2011, MNRAS, 411,2035

Navarro, J. F., Frenk, C. S., \& White, S. D. M. 1997, ApJ, 490, 493

Niemi, S.-M., Heinämäki, P., Nurmi, P., \& Saar, E. 2010, MNRAS, 405, 477

Norris, M. A., Sharples, R. M., Bridges, T., et al. 2008, MNRAS, 385, 40

Pota, V., Forbes, D. A., Romanowsky, A. J., et al. 2013, MNRAS, 428, 389

Proctor, R. N., Forbes, D. A., Romanowsky, A. J., et al. 2009, MNRAS, 398, 91

Prugniel, P., \& Heraudeau, P. 1998, A\&AS, 128, 299

Richtler, T., Schuberth, Y., Hilker, M., et al. 2008, A\&A, 478, L23

Richtler, T., Salinas, R., Misgeld, I., et al. 2011a, A\&A, 531, A119

Richtler, T., Famaey, B., Gentile, G., \& Schuberth, Y. 2011b, A\&A, 531, A100

Romanowsky, A. J., Douglas, N. G., Arnaboldi, M., et al. 2003, Science, 301, 1696

Salinas, R., Richtler, T., Bassino, L. P., Romanowsky, A. J., \& Schuberth, Y. 2012, A\&A, 538, A87

Samurović, S., \& Danziger, I. J. 2005, MNRAS, 363, 769

Sánchez-Blázquez, P., Peletier, R. F., Jiménez-Vicente, J., et al. 2006, MNRAS, 371,703

Schauer, A. T. P., Remus, R.-S., Burkert, A., \& Johansson, P. H. 2014, ApJ, 783, L32

Schuberth, Y., Richtler, T., Hilker, M., et al. 2010, A\&A, 513, A52

Schuberth, Y., Richtler, T., Hilker, M., et al. 2012, A\&A, 544, A115

Temi, P., Brighenti, F., Mathews, W. G., \& Bregman, J. D. 2004, ApJS, 151, 237

Teodorescu, A. M., Méndez, R. H., Bernardi, F., Riffeser, A., \& Kudritzki, R. P. 2010, ApJ, 721, 369

Tonry, J. L., Dressler, A., Blakeslee, J. P., et al. 2001, ApJ, 546, 681

Tortora, C., Romanowsky, A. J., \& Napolitano, N. R. 2013, ApJ, 765, 8

Tran, K.-V. H., Simard, L., Illingworth, G., \& Franx, M. 2003, ApJ, 590, 238

van Dokkum, P. G., \& Conroy, C. 2010, Nature, 468, 940

Weinmann, S. M., Kauffmann, G., van den Bosch, F. C., et al. 2009, MNRAS, 394,1213 